OPEN ACCESS

Edited by:

Armida Mucci,

University of Campania Luigi

Vanvitelli, Italy

Reviewed by:

Stefano Barlati,

University of Brescia, Italy

Ernest Marek Tyburski,

Pomeranian Medical University in

Szczecin, Poland

*Correspondence:

Janusz K. Rybakowski

janusz.rybakowski@gmail.com

Specialty section: This article was submitted to

Schizophrenia,

a section of the journal

Frontiers in Psychiatry

Received: 18 October 2021 Accepted: 08 December 2021

Published: 24 December 2021

Citation:

Wójciak P, Domowicz K, Zabłocka M,

Michalak M and Rybakowski JK

(2021) Association of Negative

Symptoms of Schizophrenia Assessed by the BNSS and SNS Scales With

Neuropsychological Performance: A

Gender Effect.

Front. Psychiatry 12:797386

doi: 10.3389/fpsyt.2021.797386

\section{Association of Negative Symptoms of Schizophrenia Assessed by the BNSS and SNS Scales With Neuropsychological Performance: A Gender Effect}

\author{
Paweł Wójciak ${ }^{1}$, Klaudia Domowicz ${ }^{1}$, Marta Zabłocka ${ }^{1}$, Michał Michalak ${ }^{2}$ and \\ Janusz K. Rybakowski ${ }^{1 *}$
}

${ }^{1}$ Department of Adult Psychiatry, Poznan University of Medical Sciences, Poznan, Poland, ${ }^{2}$ Department of Computer Science and Statistics, Poznan University of Medical Sciences, Poznan, Poland

Objective: The relationship between negative symptoms and neurocognitive performance in schizophrenia is well documented, but the mechanism of these connections remains unclear. The study aims to measure the relationship between the results on the new scales for the assessment of negative symptoms such as Brief Negative Symptom Scale (BNSS) and Self-evaluation of Negative Symptoms (SNS), and the results of some neurocognition tests. The second aim is to assess a possible gender effect on these associations.

Methods: The study included 80 patients (40 men, 40 women) with schizophrenia, aged 19-63 (mean 38 years), during the improvement period (total PANSS score $<80$, unchanged pharmacological treatment in the last 3 weeks). They were assessed using the BNSS, SNS, Personal and Social Performance (PSP) scales, and the tests for neuropsychological performance such as the Trail Making Test (TMT-A, TMT-B), Stroop Color-Word Interference Test, Verbal fluency tests (VFT), Category fluency test (CFT), and Digit Symbol Substitution Test (DSST).

Results: Male patients obtained higher scores than females on some PANSS and BNSS items. No gender differences were observed for the SNS scale. Female patients scored better in the PSP and CFT. In male patients, a significant positive correlation between the intensity of negative symptoms measured by the BNSS and the results of PSP with the Trail Making Test was observed. In female patients, we found a positive correlation between the results of BNSS and PSP with the Stroop Color-Word Interference Test.

Conclusion: The obtained results confirm the relationship between negative symptoms and neurocognition in schizophrenia patients. However, in male and female patients such association was observed for different cognitive domains. Further research is needed to explain the nature of these differences.

Keywords: self-evaluation of negative symptoms, negative symptoms, neurocognition, brief negative symptom scale, schizophrenia 


\section{INTRODUCTION}

Schizophrenia is a multidimensional disorder, the disease syndrome consists of positive, negative, disorganized, and affective symptoms with varying degrees of intensity, accompanied by disorders of neurocognition and social cognition. Many studies focus on assessing the mutual relations between these domains. The relationship between negative symptoms and cognitive impairment is well documented (1), it seems to be stronger (2) than for positive (3) and affective symptoms (4). The mechanism of these connections, however, remains unclear (5), the obtained results are heterogeneous, and there are also methodological controversies.

Negative symptoms and cognitive impairment share many things in common. Their frequency, course, prognostic significance, and correlation with many aspects of daily functioning are similar (6). In most studies, the severity of both disorders negatively correlates with each other $(7,8)$. It should be noted, however, that there are also studies in which no correlation between negative symptoms and neurocognition was found $(9,10)$.

To explain these discrepancies, Harvey et al. (6) suggest the possibility of the occurrence of as many as four different models of the relationship between negative symptoms and cognitive impairment. Both disorders are a common dimension of the disease, both disorders are a separate dimension but with a similar etiology, each disorder has a separate etiology but with some common elements, and finally both disorders are a separate dimension and have a separate etiology. Summing up, the authors support the conclusion that both symptoms are separate, a similar position is taken by Yolland et al. (11). In their work, they did not observe the relationship between the severity of negative symptoms and cognitive impairment, which led them to suggest that negative symptoms and neurocognition are separate constructs and require separate therapeutic strategies.

Despite the above reservations, it seems that the mutual relationship between these two symptoms is more complex and multidimensional. Harvey et al. (6) indicate that cognitive disorders appear even before the clinical onset of psychosis and, similarly to negative symptoms, can be referred to as "early symptoms," which allows treating them as a neurodevelopmental component of schizophrenia. Ventura et al. (7) point out that negative symptoms may mediate between cognitive impairment and functional outcome, for Velligan et al. (12) negative symptoms are a behavioral consequence of cognitive impairment.

Harvey et al. (6) also indicate that the relationship between negative symptoms and cognitive impairment depends on the definition of negative symptoms while emphasizing that negative symptoms are defined based on the clinical picture, and cognitive disorders based on the tests performed. Additionally, the definition of negative symptoms differs depending on whether we are dealing with primary or secondary negative symptoms. Thus, patients with stable negative symptoms forming the deficit syndrome are characterized by a significant intensification of cognitive disorders (13).

As indicated by Milev et al. (2), the type of research tool used has a significant impact on the obtained results. In the research so far, the most frequently used scale is the Positive and Negative Syndrome Scale (PANSS). Some symptoms of cognitive nature (deficits in abstract thinking, stereotyped thinking, poor attention) are defined as negative or as general symptoms of schizophrenia by the PANSS. SANS also describes behavioral disorders such as deficits in social, occupational, and educational activity as negative symptoms, the same problem applies to attention disorders treated as negative symptoms (6). In a large systematic review of studies assessing the relationship of schizophrenia symptoms, including negative ones, with cognitive impairment, the authors cited the results of 18 studies from 2008-2019, none of the research protocols included the so-called 2nd generation scales for the assessment of negative symptoms, taking into account the NIMH- MATRICS consensus statement on negative symptoms $(14,15)$.

The nature and structure of the scales used so far lead, as noted by Alpert et al. (16) to evaluate individual items of negative symptoms through the prism of the global assessment of the clinical picture, which significantly affects the obtained results.

It also seems important to consider sex differences in research protocols that affect the age of onset of the disease, premorbid adjustment, course, and expression of clinical symptoms, which is particularly important in the context of the assessment of cognitive disorders and their relationship with negative symptoms (17).

Significant gender effects have been observed in schizophrenia for both negative symptoms and neurocognition. Moriarty et al. (18) suggested that negative symptom severity is greater in male patients. This was confirmed in subsequent research of Galderisi et al. (19) as well as our recent study as to the assessment on the BNSS scale (20).

The results of studies assessing the relationship between gender and cognitive processes in patients with schizophrenia are inconclusive. Some studies have reported superior cognitive function in women, others found the opposite or no gender difference, while some observed a reversal of normal sexual dimorphism (21). In numerous studies, men with schizophrenia performed worse in terms of cognitive functions, both in comparison with the control group (22) and female patients $(23,24)$. Only a few studies have shown better neurocognitive performance in men compared to women with schizophrenia $(25,26)$. There are also studies showing no difference in terms of neurocognition between men and women with schizophrenia $(27,28)$. As a factor assisting better cognitive performance in women with schizophrenia, estrogen and its neuroprotective role in the body are most often indicated $(17,29)$.

It seems that the endocrine system and its influence on the central nervous system are more closely related to the development of schizophrenia and sexual differences in the course of this disease than previously thought (30). Recent studies show that structural plasticity of the brain is regulated by hormones (31), not only in the systemic aspect, but also in the local-the brain is also capable of locally generating estrogens, either from androgens and possibly also directly from cholesterol $(32,33)$. The estrogen hypothesis is supported by late-onset age and second incidence peak around menopausal age in women. Indeed, estrogen deficiency is highly related 
to the severity of psychiatric symptoms in women during menopause (34). For example, female schizophrenia patients often have more severe symptoms in the low estrogen phase of their menstrual cycle (35). Interestingly, the negative correlation between plasma estrogen levels and schizophrenia symptoms was also reported in male patients (36). The biochemical nature of the neuroprotective effects of estrogens has not been fully identified yet, but a number of studies points to a direct implication of the dopaminergic system, in addition to glutamate and GABA (37). When it comes to testosterone, studies found that low levels of testosterone appear to be associated with more severe symptoms, although results are less consistent than for estrogens (38). For instance, studies found that schizophrenia patients with low levels of testosterone often have predominantly negative symptoms, and serum testosterone levels are associated with greater severity of negative symptoms (39). Another important hormone associated with the pathogenesis of schizophrenia appears to be oxytocin. Several studies suggest that schizophrenia patients with higher levels of plasma oxytocin develop fewer psychotic symptoms (40), and have better cognition (41). Oxytocin is thought to regulate central dopamine, and might therefore exhibit antipsychotic effects (42).

The study aims to evaluate the relationship between negative symptoms assessed by the so-called 2nd generation scales such as the Brief Negative Symptoms Scale (BNSS) (43), and the Self-evaluation of Negative Symptoms (SNS) (44), with neuropsychological performance, in patients with chronic schizophrenia. The hypothesis was put forward postulating that such a relationship can be connected with gender.

\section{METHODS}

\section{Study Design and Participants}

This is a cross-sectional study. The study participants were recruited among subjects attending the outpatient and inpatient unit of the Department of Adult Psychiatry, Poznan University of Medical Sciences. The recruitment was carried out from October 31, 2016, to July 15, 2017.

Eighty patients (40 male, 40 female), aged 19-63 (mean $38 \pm 11$ ) years, with a diagnosis of schizophrenia, according to ICD-10 and DSM-5 were included. Their onset of illness was $26 \pm 8$ years, the duration of illness was $12 \pm 9$ years, and the number of hospitalization was $6 \pm 6$. They were either inpatients (60 subjects) or were under the care of the outpatient clinic (20 subjects), at the Department of Adult Psychiatry, Poznan University of Medical Sciences. All patients remained in the phase of symptomatic stabilization defined as achieving a total PANSS score of the maximum of 80 points. They received unchanged pharmacological treatment in the last 3 weeks (inpatients) or 3 months (outpatients). Four male and four female patients were treated with haloperidol. The remaining subjects received atypical antipsychotics (olanzapine, clozapine, aripiprazole, risperidone, quetiapine, amisulpride, and ziprasidone) the proportion of which was comparable in male and female patients.
Participants with significant physical, visual, verbal impairments, neurological disorders, and substance abuse or dependence, were excluded.

The local Ethics Committee approved the study (Agreement no $1122 / 16$ ), and it was performed in accordance with the ethical standards of the Declaration of Helsinki (45). All participants signed a written informed consent to participate in the study, after receiving a detailed explanation of the study's procedures and goals.

\section{Neuropsychological and Clinical Assessment \\ General Psychopathological Assessment}

The Positive and Negative Syndrome Scale (PANSS) PANSS was developed by Kay et al. (46). It is a 30 -item rating scale developed specifically to assess patients with schizophrenia. It is divided into three subscales, a positive scale with seven positive symptoms (P1-P7), a negative scale with seven negative symptoms (N1N7), and a general psychopathology scale with 16 items (G1G16). Psychopathology severity is defined as $1=$ absent; $2=$ minimal; $3=$ mild; $4=$ moderate; $5=$ moderate-severe; $6=$ severe; $7=$ extreme.

\section{Assessment of Negative Symptoms The Brief Negative Symptoms Scale}

The BNSS was developed by Kirkpatrick et al. (43). The scale defines negative symptoms as the absence or decrease in behaviors and subjective experiences that are normally present in a person from the same culture and age group. The scale has 13 items organized into six subscales: anhedonia (intensity and frequency of pleasure, the intensity of expected pleasure), distress (subject's experience of unpleasant or distressing emotion of any kind, such as sadness, depression, anxiety, grief, anger), asociality (reported as reduced social activity accompanied by decreased interest in forming close relationships with othersbehavior, internal experience), avolition (reported as a reduction in the initiation of and persistence in activity-behavior, internal experience), blunted affect (which refers to a decrease in the outward expression of emotion-facial expression, vocal expression, gestures), and alogia (reported as poverty of speechthe quantity of speech, spontaneous elaboration of speech). The examination is of an interview nature, based on a manual including, among other things, prompts and suggested questions. All items are rated on a 7 -point scale (0-6), with anchor points ranging from a symptom being absent (0) to severe (6). The time frame for the ratings is 1 week. The basis for the interview is provided by the patient-participant observation also constitutes an important element-or, if needed, data obtained from external sources. The Polish version of BNSS was used in the study (47).

\section{The Self-Evaluation of Negative Symptoms}

The SNS was developed by Dollfus et al. (44). The scale evaluates all five groups of negative symptoms, i.e., social withdrawal, blunted affect, avolition, anhedonia, and alogia. Social withdrawal (asociality) assesses social relationships as well as the patient's propensity to establish new relationships; diminished emotional range (blunted affect) reflects a presence 
of happiness or sadness in situations in which they are usually felt; avolition relates to motivation, energy level and the ability to achieve goals; anhedonia describes the experienced and expected pleasure; alogia (poverty of speech) is evaluated by the subjective assessment of the examined person. The scale contains 20 items, four items for each negative symptom, and the evaluation pertains to the previous week. For maximal simplification, the number of responses was limited to 3: "strongly agree" scoring 2 , "somewhat agree" scoring 1, and "strongly disagree" scoring 0 . The total score is the sum of the 20 items, ranging from 0 (no negative symptoms) to 40 (severe negative symptoms). The Polish version of SNS was used in the study (48).

\section{Assessment of Social Functioning}

The Personal and Social Performance scale (PSP) evolved based on the social functioning component of the DSM-IV Social and Occupational Functioning Assessment Scale (SOFAS) as an effort to assess social functioning in schizophrenia. It is being proposed as an improvement over the Global Assessment of Functioning (GAF) scale and SOFAS. PSP is a 100-item scale, divided into 10 similar intervals. The score is based on the assessment of patient's performance in four categories: socially useful activities, personal and social relationships, self-care, disturbing and aggressive behavior. PSP provides a score between 1 and 100, divided into 10 equal intervals to rate the degree of difficulty. Higher scores represent better personal and social functioning (49).

\section{Assessment of Neurocognition}

\section{Trail Making Test (TMT-A, TMT-B)}

Trail Making Test is used to assess working memory and as an indicator of visual scanning, graphomotor speed, and executive function. In part $\mathrm{A}$, the subject is asked to connect the circles marked with numbers from 1 to 25 as quickly as possible, which allows the measurement of psychomotor speed. In part B, the subject's task is to as quickly as possible connect the circles marked with numbers 1-13 and letters A-L in the order 1-A-2-B$3-\mathrm{C}$, etc. This operation requires keeping two different sequences of numbers and letters in working memory. The result of the test is the time and correctness of parts A and B of the test (50). We also calculated the TMT B-A difference as proposed in recent studies (51).

\section{Stroop Color-Word Interference}

Test Stroop Color-Word Interference Test is used to evaluate verbal working memory. The test consists of two parts. In the first one (Reading Color Names in Black, RCNb, Stroop A), the respondent is asked to read the words (color names) written in black on a white sheet as soon as possible. In the second part (Naming Color of Word different, NCWd, Stroop B), the respondent's task is to name the font color of individual words, whereby the font color of the word is different from the color indicated by him. This activity requires a change in the form of reaction (switching from content to color). The result of the test is the time needed to complete the first and second parts and the number of incorrect answers (52). The difference between Stroop B-A was also calculated (53).

\section{Verbal Fluency Tests and Category Fluency Test}

Verbal fluency tests and Category fluency test are used to evaluate speech functions, and semantic memory access and executive functions. The tests assess the ability to pronounce words fluently in accordance with a specific criterion in a given time (for 60 seconds). This criterion is a given letter (in VFT) or category (in CFT). In the Polish version used in the study, in the part assessing verbal fluency, the respondent's task is to list for $1 \mathrm{~min}$ as many words starting with the letters of the alphabet as possible: $\mathrm{K}, \mathrm{O}, \mathrm{S}$, and in the part assessing categorical fluency, the greatest number of words from three categories: animals, vegetables, fruits $(54,55)$.

\section{Digit Symbol Substitution Test}

Digit Symbol Substitution Test is a wordless test on the Wechsler Adult Intelligence Scale (WAIS-R). It is used to evaluate motor speed, attention, and visuoperceptual functions. The task of the examined person is to assign symbols to digits according to a given key within a specified time $(90 \mathrm{~s})$. The result of the test is the number of correctly assigned symbols $(56,57)$.

\section{Statistics}

Continuous data is presented as means and standard deviations. Except of the descriptive statistics the effect size was denoted (d Cohen). The Shapiro-Wilk test was applied to check if data follow the normal distribution. Because the data of BNSS, SNS, and PSP were consistent with a normal distribution, the gender differences were assessed by the two-tailed $t$-test. The data of neurocognitive tests was not normally distributed which is why the BoxCox transformation of non-normal dependent variables was performed in order to assess a normal shape. Additionally, the Benjamini-Hochberg adjusted $p$-value for multiple comparisons was calculated. Statistical relationships between BNSS, SNS, PSP and the results of neurocognitive tests were calculated using Pearson correlation coefficient. The statistical analysis was performed with the use of TIBCO Software Inc. (2017). Statistica (data analysis software system), version 13. http://statistica.io. All test were considered significant at $p<0.05$.

\section{RESULTS}

The mean age of women with schizophrenia was higher than men (41 vs. $35, p=0.048$ ), the mean number of years of education was greater in the group of women than in men (13 vs $12, p=0.046$ ). There was no gender difference in disease duration and number of hospitalizations (see Table 1).

The comparison of the results of psychometric tests in male and female schizophrenia patients is shown in Table 2.

Among the PANSS scores, there was a significantly higher score on negative symptoms and total score in male patients (both $p<0.05$, d Cohen 0.6, medium effect size). On the BNSS scale, there was a significantly higher score of male patients in subscales of distress ( $p=0.01, \mathrm{~d}$ Cohen 0.7$)$ and asociality ( $p=$ 0.05 , d Cohen 0.6). There was also a tendency ( $p<0.1, \mathrm{~d}$ Cohen $0.5)$ for the higher total BNSS score. No gender differences were found as to the assessment on the SNS scale. 
Female patients scored significantly better on the PSP scale (68.1 vs. $60.7, p=0.021$, d Cohen 0.5 )

The comparison of the results of neurocognition tests in male and female schizophrenia patients is shown in Table 3.

No significant gender differences were observed except that female patients performed significantly better on the Category Fluency Test $(p<0.001, \mathrm{~d}$ Cohen 1.0).

The relationships between the results of women and men on the BNSS, SNS, and PSP scales and the results of neurocognitive tests are shown in Table 4.

In male patients, we found a significant positive correlation between BNSS total and subscales and a significant negative correlation between the results on PSP with the Trail Making Test (TMT-A, TMT-B).

TABLE 1 | Demographic characteristics of the study group.

\begin{tabular}{lcccc}
\hline Category & $\begin{array}{c}\text { Total } \\
\boldsymbol{n}=\mathbf{8 0}\end{array}$ & $\begin{array}{c}\text { Women } \\
\boldsymbol{n}=\mathbf{4 0}\end{array}$ & $\begin{array}{c}\text { Men } \\
\boldsymbol{n}=\mathbf{4 0}\end{array}$ & $\boldsymbol{p}$ \\
\hline $\begin{array}{l}\text { Age } \\
\text { Mean } \pm \text { SD (years) }\end{array}$ & $38 \pm 10$ & $40 \pm 12$ & $35 \pm 8$ & $\mathbf{0 . 0 4 8}^{*}$ \\
$\begin{array}{l}\text { Years of education } \\
\text { Mean } \pm \text { SD }\end{array}$ & $12 \pm 2$ & $13 \pm 2$ & $12 \pm 2$ & $\mathbf{0 . 0 4 6 ^ { * }}$ \\
$\begin{array}{l}\text { IIness duration } \\
\text { Mean } \pm \text { SD (years) }\end{array}$ & $12 \pm 9$ & $13 \pm 9$ & $10 \pm 9$ & 0.090 \\
$\begin{array}{l}\text { Number of hospitalizations } \\
\text { Mean } \pm \text { SD }\end{array}$ & $6 \pm 6$ & $6 \pm 6$ & $6 \pm 6$ & 0.785 \\
& & & & \\
\hline
\end{tabular}

${ }^{*}$ Difference between men and women significant $p<0.05$.
In female patients, a significant positive correlation between the intensity of negative symptoms measured by the BNSS total and most subscales, and the results of Stroop B was found. The BNSS asociality correlated with the results of Stroop A. The intensity of negative symptoms measured by the SNS sum, social withdrawal, and anhedonia subscale correlated with the results of Stroop A. A significant negative correlation between the results of PSP with the results of both Stroop A and B was observed.

\section{DISCUSSION}

The results obtained may indicate gender differences in schizophrenia patients in the assessment of negative symptoms and personal and social performance as well as in the correlations between negative symptoms and the results of neurocognitive tests. In male patients, the higher score on BNSS and worse results on PSP make a corroboration of our previous work (58). Also, the better results in schizophrenic women on PSP were recently confirmed by Spanish investigators (59).

The main aim of our study was to investigate the correlation between negative symptoms and neurocognitive functions. Such correlation of negative symptoms with various indexes of neurocognition has been described in many papers $(5,6,60)$. In all these studies, the negative symptoms were assessed by the PANSS. However, in a recent validation of the BNSS in Korean patients with schizophrenia, a correlation of negative symptoms assessed by this scale with neurocognitive tests of verbal memory, processing speed, and attention was found (61).

TABLE 2 | The comparison of the results of clinical and psychometric tests in female and male schizophrenia patients.

\begin{tabular}{|c|c|c|c|c|c|c|c|c|c|}
\hline \multirow[t]{2}{*}{ Parameter } & \multicolumn{2}{|c|}{ Total } & \multicolumn{2}{|c|}{ Women } & \multicolumn{2}{|c|}{ Men } & \multirow{2}{*}{$\frac{\text { Effect size }}{\text { d Cohen }}$} & \multirow[t]{2}{*}{$p$-value } & \multirow[t]{2}{*}{ Adjusted $p$-value ${ }^{*}$} \\
\hline & Mean & SD & Mean & SD & Mean & SD & & & \\
\hline PANSS positive & 13.6 & 3.1 & 12.8 & 3.1 & 14.3 & 3 & -0.5 & 0.041 & 0.082 \\
\hline PANSS negative & 16.6 & 5 & 15.1 & 4.9 & 18.2 & 4.8 & -0.6 & 0.006 & $0.022^{*}$ \\
\hline PANSS general & 29 & 5.3 & 28.2 & 6.2 & 29.7 & 4.3 & -0.3 & 0.221 & 0.221 \\
\hline PANSS sum & 59.2 & 10.8 & 56.2 & 11.7 & 62.2 & 8.8 & -0.6 & 0.012 & $0.035^{\star}$ \\
\hline BNSS anhedonia & 6.2 & 3.4 & 5.6 & 3.7 & 6.6 & 3.1 & -0.3 & 0.156 & 0.156 \\
\hline BNSS distress & 1.3 & 1 & 1 & 1 & 1.7 & 1 & -0.7 & 0.001 & $0.010^{*}$ \\
\hline BNSS asociality & 3.9 & 2 & 3.3 & 2 & 4.5 & 1.9 & -0.6 & 0.009 & $0.050^{\star}$ \\
\hline BNSS avolition & 4.1 & 2.1 & 3.7 & 2.1 & 4.6 & 2 & -0.4 & 0.086 & 0.156 \\
\hline BNSS blunted affect & 4.9 & 3.2 & 4.2 & 3.2 & 5.7 & 3 & -0.5 & 0.038 & 0.153 \\
\hline BNSS alogia & 2.6 & 2.2 & 2.1 & 2 & 3 & 2.4 & -0.4 & 0.086 & 0.156 \\
\hline BNSS sum & 23.3 & 11.5 & 20.2 & 11.7 & 26.4 & 11.6 & -0.5 & 0.020 & 0.099 \\
\hline SNS social withdrawal & 2.9 & 2 & 2.5 & 2.1 & 3.3 & 1.8 & -0.4 & 0.076 & 0.455 \\
\hline SNS diminished emotion & 3.4 & 2.1 & 3 & 2.1 & 3.7 & 2 & -0.3 & 0.144 & 0.499 \\
\hline SNS alogia & 3.8 & 2.4 & 3.6 & 2.6 & 4 & 2.3 & -0.2 & 0.499 & 0.499 \\
\hline SNS avolition & 3.8 & 2.3 & 3.5 & 2.5 & 4 & 2 & -0.2 & 0.388 & 0.499 \\
\hline SNS anhedonia & 2.8 & 2.4 & 2.5 & 2.5 & 3.1 & 2.4 & -0.2 & 0.301 & 0.499 \\
\hline SNS sum & 16.7 & 8.8 & 15.2 & 9.9 & 18.2 & 7.4 & -0.3 & 0.144 & 0.499 \\
\hline PSP & 60.4 & 14.5 & 68.1 & 14.1 & 60.7 & 14.1 & 0.5 & 0.021 & $0.021^{*}$ \\
\hline
\end{tabular}

*Difference between men and women significant $p \leq 0.05$. Significant differences are marked in bold. 
TABLE 3 | The comparison of the results of neurocognition tests in female and male schizophrenia patients.

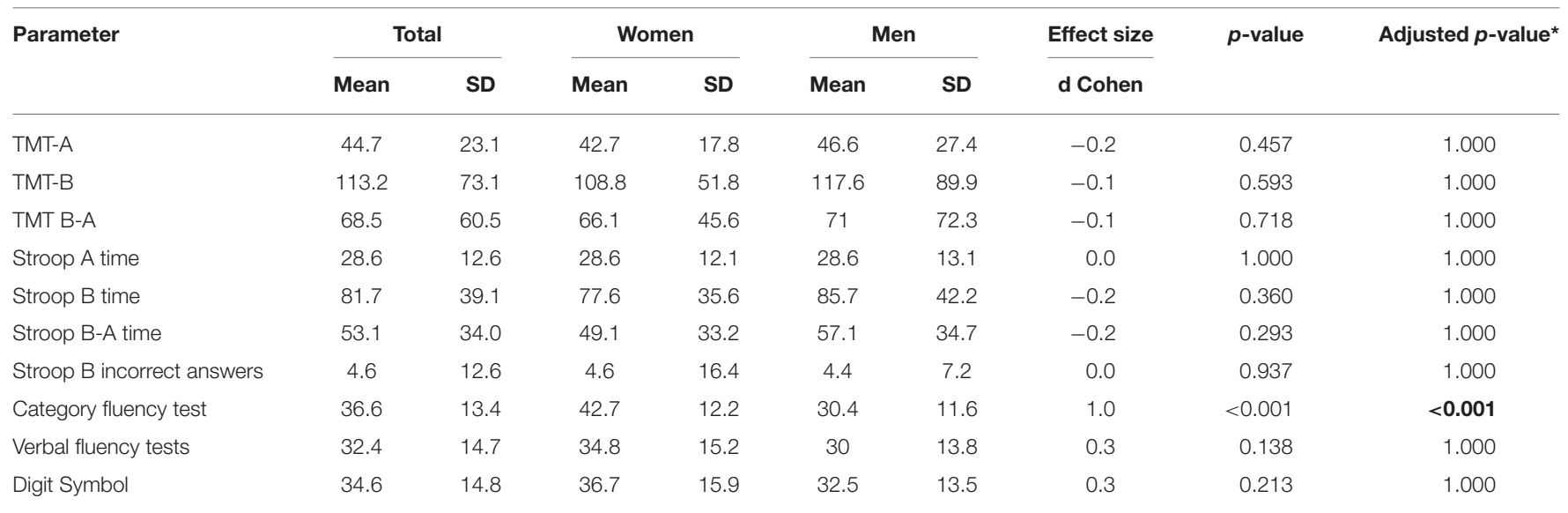

${ }^{\star}$ Difference between men and women significant $p<0.001$. Significant differences are marked in bold.

TABLE 4 | Relationships between the results of men and women on the BNSS, SNS, and PSP scale and the results of neurocognition tests.

\begin{tabular}{|c|c|c|c|c|c|c|c|c|}
\hline \multirow[t]{2}{*}{ Parameter } & \multicolumn{4}{|c|}{ Male } & \multicolumn{4}{|c|}{ Female } \\
\hline & TMT-A & TMT-B & Stroop A & Stroop B & TMT-A & TMT-B & Stroop A & Stroop B \\
\hline BNSS anhedonia & $\begin{array}{c}0.399 \\
p=0.011\end{array}$ & $\begin{array}{c}0.328 \\
p=0.039\end{array}$ & 0.127 & 0.179 & 0.027 & -0.203 & 0.306 & $\begin{array}{c}0.368 \\
p=0.019\end{array}$ \\
\hline BNSS asociality & $\begin{array}{c}0.343 \\
p=0.03\end{array}$ & $\begin{array}{c}0.359 \\
p=0.023\end{array}$ & 0.209 & 0.207 & -0.099 & -0.250 & $\begin{array}{c}0.380 \\
p=0.015\end{array}$ & 0.3 \\
\hline BNSS avolition & $\begin{array}{c}0.459 \\
p=0.003\end{array}$ & $\begin{array}{c}0.356 \\
p=0.024\end{array}$ & 0.18 & 0.257 & -0.053 & -0.284 & 0.308 & $\begin{array}{c}0.405 \\
p=0.009\end{array}$ \\
\hline BNSS blunted affect & $\begin{array}{c}0.524 \\
p=0.001\end{array}$ & $\begin{array}{c}0.398 \\
p=0.011\end{array}$ & -0.026 & 0.225 & 0.07 & -0.255 & 0.24 & $\begin{array}{c}0.509 \\
p=0.001\end{array}$ \\
\hline BNSS alogia & $\begin{array}{c}0.523 \\
p=0.001\end{array}$ & $\begin{array}{c}0.414 \\
p=0.008\end{array}$ & 0.284 & 0.266 & 0.079 & -0.216 & 0.24 & $\begin{array}{c}0.585 \\
p=0.001\end{array}$ \\
\hline BNSS sum & $\begin{array}{c}0.502 \\
p=0.001\end{array}$ & $\begin{array}{c}0.406 \\
p=0.009\end{array}$ & 0.158 & 0.254 & 0.009 & -0.272 & 0.311 & $\begin{array}{c}0.487 \\
p=0.001\end{array}$ \\
\hline SNS social withdrawal & 0.165 & 0.263 & 0.007 & 0.011 & -0.114 & -0.113 & $\begin{array}{c}0.432 \\
p=0.005\end{array}$ & 0.148 \\
\hline SNS diminished emotion & 0.285 & 0.225 & 0.003 & 0.099 & 0.013 & -0.166 & 0.33 & 0.35 \\
\hline SNS alogia & 0.142 & 0.069 & 0.216 & 0.005 & 0.038 & -0.153 & 0.337 & 0.129 \\
\hline SNS avolition & -0.715 & 0.208 & 0.067 & -0.037 & 0.004 & -0.013 & 0.166 & 0.21 \\
\hline SNS anhedonia & 0.215 & 0.209 & -0.175 & 0.001 & 0.026 & -0.055 & $\begin{array}{c}0.388 \\
p=0.013\end{array}$ & 0.126 \\
\hline SNS sum & 0.218 & 0.275 & 0.029 & 0.019 & -0.001 & -0.097 & $\begin{array}{c}0.391 \\
p=0.012\end{array}$ & 0.23 \\
\hline PSP sum & $\begin{array}{c}-0.439 \\
p=0.005\end{array}$ & $\begin{array}{c}-0.343 \\
p=0.03\end{array}$ & -0.163 & -0.245 & -0.001 & 0.124 & $\begin{array}{c}-0.315 \\
p=0.047\end{array}$ & $\begin{array}{c}-0.367 \\
p=0.02\end{array}$ \\
\hline
\end{tabular}

Pearson correlation coefficient and " $p$ " of significant correlations were given.

Recently, Eack and Keshavan (62) suggested three potential underpinnings of such correlation. First, that cognitive and negative symptoms share similar underlying pathophysiology giving rise to their overlap and impact on functioning. Second, that cognitive impairment leads to greater negative symptoms and reduced functional performance, and third, that negative symptoms lead to impaired cognitive abilities that drive poor functional outcomes. In this respect, is of importance the paper of Luther et al. (63) in which in the 20-year longitudinal study, it was found that greater negative symptoms predicted reduced neurocognition and indirectly-impaired work functioning.

In our research, the results of the neurocognitive tests were numerically worse in males, however, the only significant difference was in the category fluency. Whereas, significant correlations between negative symptoms and the results on some neurocognitive tests were obtained. However, in male and female 
patients they were observed for different cognitive tests. In males, a significant positive correlation between the intensity of negative symptoms measured by the BNSS and the Trail Making Test was observed. In female patients, we found positive correlations between the results of the BNSS and Stroop ColorWord Interference Test. Interestingly, similar correlations in male and female schizophrenia patients were obtained between these neurocognitive tests and the results of the PSP scale.

Therefore, the most intriguing result of our study is a relationship obtained in male and female schizophrenia patients between negative symptoms and social functioning, with different cognitive domains. In men, increased negative symptoms and worse functioning were correlated with inferior results of TMT test measuring visual attention and task switching, while in female patients such correlations were obtained with a worse outcome of the Stroop test, assessing the ability to inhibit cognitive interference. Probably, it can be speculated that these clinical and functioning disturbances are mostly underpinned by different cognitive factors depending on gender.

Why impaired cognitive abilities involved in the Trail Making Test are related to the intensity of negative symptoms and social performance in men but not in women? It can be speculated that in men, impaired psychomotor speed, attention, and mental flexibility are more important than in women for a generation of negative symptoms and social functioning. A similar question can be asked, why impaired performance on the Stroop test is related to the intensity of negative symptoms and social performance in women but not in men? Probably, in women, impaired ability to inhibit cognitive interference is more important than in men for a generation of negative symptoms and social functioning.

Regardless of the neurophysiological mechanisms of these differences, the results may point to distinctive features of negative symptoms in male and female schizophrenia patients. This was also shown in our recent study in which in female patients, a significant positive correlation between the intensity of negative symptoms measured by the BNSS scale and the concentration of high-density lipoprotein (HDL) cholesterol, and a trend for negative correlation with BMI was observed. Whereas, such correlations were not found in male patients (58).

The study suffers from four major limitations. First, the experimental sample consisted of chronic and clinically stable subjects with schizophrenia and did not include a control group (e.g., healthy controls). Second, we did not include the measure of IQ, which is important for cognitive functioning in schizophrenia and potential differences between groups. Third, the study took place in a controlled environment, which resulted in a low ecological validity of the used neuropsychological assessment, consequently, it is more difficult to generalize the study's findings. Last, but not least, the chlorpromazine equivalents of drugs that were administered to patients and which may have an important role for cognitive functioning in schizophrenia were not recorded in the study. Given the importance of the above limitations, future research should take them into account.

In conclusion, the results obtained confirm the relationship between negative symptoms and neurocognition in schizophrenia patients, although for different cognitive domains, depending on gender. Considering the above aspects together seems to be important from a clinical point of view, as suggested by Li et al. (34). Consideration of gender differences in schizophrenia provides an important insight for understanding the sex-specific characteristics of the disease's onset, symptoms, and the opportunity to deliver sex-specific treatments and care for schizophrenia. The above observations have already been reflected in previous studies in which an integrated therapeutic approach with the combination of neurocognition and social cognition might be a more effective approach to treating the symptomatology of people with schizophrenia $(1,64)$.

Further research is needed to confirm these findings and to explain the nature of these differences. It seems particularly important to correctly define negative symptoms, to standardize tools for the assessment of neurocognition, and in the aspect of gender differences-to take into account such differentiating factors as the endocrine system or molecular-genetic findings. Study design should also take into account ecological validity in subsequent studies, to predict better behaviors in realworld settings.

\section{DATA AVAILABILITY STATEMENT}

The original contributions presented in the study are included in the article/supplementary material, further inquiries can be directed to the corresponding author.

\section{ETHICS STATEMENT}

The studies involving human participants were reviewed and approved by Bioethics Committee, Poznan University of Medical Sciences, Poznan, Poland. The patients/participants provided their written informed consent to participate in this study.

\section{AUTHOR CONTRIBUTIONS}

PW took part in the design of the study and wrote the first draft of the manuscript. KD performed psychometric assessments. MZ performed neurocognitive assessments. MM performed statistical calculation. JR elaborated the design of the study and wrote the final version of the manuscript. All authors accepted the final version of the manuscript.

\section{FUNDING}

The paper was helped by own funds of the Department of Adult Psychiatry, Poznan University of Medical Sciences. 


\section{REFERENCES}

1. Lam BYH, Raine A, Lee TMC. The relationship between neurocognition and symptomatology in people with schizophrenia: social cognition as the mediator. BMC Psychiatry. (2014) 14:138. doi: 10.1186/1471-244X-14-138

2. Milev P, Ho BC, Arndt S, Andreasen NC. (2005). Predictive values of neurocognition and negative symptoms on functional outcome in schizophrenia: a longitudinal first-episode study with 7-year follow-up. Am J Psychiatry. (2005) 162:495-506. doi: 10.1176/appi.ajp.162.3.495

3. Ventura J, Wood RC, Hellemann GS. Symptom Romains and neurocognitive functioning can help differentiate social cognitive process in schizophrenia: a meta-analysis. Schizophr Bull. (2011) 39:102-11. doi: 10.1093/schbul/sbr067

4. Smith TE, Hull JW, Goodman M, Hedayat-Harris A, Willson DF, Israel LM, et al. The relative influences of symptoms, insight, and neurocognition on social adjustment in schizophrenia and schizoaffective disorder. J Nerv Ment Dis. (1999) 187:102-8. doi: 10.1097/00005053-199902000-00006

5. Lin CH, Huang CL, Chang YC, Chen PW, Lin CY, Tsai GE. Clinical symptoms, mainly negative symptoms, mediate the influence of neurocognition and social cognition on functional outcome of schizophrenia. Schizophr Res. (2013) 146:231-7. doi: 10.1016/j.schres.2013.02.009

6. Harvey PD, Koren D, Reichenberg A, Bowie CR. Negative symptoms and cognitive deficits: what is the nature of their relationship? Schizophrenia Bulletin. (2006) 32:250-8. doi: 10.1093/schbul/sbj011

7. Ventura J, Hellemann GS, Thames AD, Koellner V, Nuechterlein KH. Symptoms as mediators of the relationship between neurocognition and functional outcome in schizophrenia: a meta-analysis. Schizophrenia Research. (2009) 111:189-99. doi: 10.1016/j.schres.2009.03.035

8. Dominguez Md G, Viechtbauer W, Simons, CJ, van Os J, Krabbendam L. Are psychotic psychopathology and neurocognition orthogonal? a systematic review of their associations. Psychological Bulletin. (2009) 135:157-71. doi: 10.1037/a0014415

9. Altamura AC, Caletti E, Paoli RA, Cigliobianco M, Zugno E, Grillo P. Correlation between neuropsychological and social cognition measures and symptom dimensions in schizophrenic patients. Psychiatry Research. (2015) 230:172-80. doi: 10.1016/j.psychres.2015.08.034

10. Bismark AW, Thomas ML, Tarasenko M, Shiluk AL, Rackelmann SY, Young JW. Relationship between effortful motivation and neurocognition in schizophrenia. Schizophr Res. (2018) 193:69-76. doi: 10.1016/j.schres.2017.06.042

11. Yolland COB, Carruthers SP, Toh WL, Neill E, Sumner PF, Thomas EHX. The relationship between negative symptoms and both emotion management and non-social cognition in schizophrenia. Spectrum disorders. J Int Neuropsychol Soc. (2020) 1-13. doi: 10.1017/S1355617720001290

12. Velligan DI, Mahurin RK, Diamond PL, Hazleton BC, Eckert SL, Miller AL. The functional significance of symptomatology and cognitive function in schizophrenia. Schizophr Res. (1997) 25:21-31. doi: 10.1016/S0920-9964(97)00010-8

13. Lysaker PH, Bell MD, Bioty SM, Zito WS. Cognitive impairment and substance abuse history as predictors of the temporal stability of negative symptoms. J Nerv Ment Dis. (1997) 185:21-6. doi: 10.1097/00005053-199701000-00004

14. Habtewold TD, Rodijk LH, Liemburg EJ, Sidorenkov G, Boezen HM, Bruggeman R. A systematic review and narrative synthesis of data-driven studies in schizophrenia symptoms and cognitive deficits. Transl Psychiatry. (2020) 10:244. doi: 10.1038/s41398-020-00919-x

15. Kirkpatrick B, Fenton WS, Carpenter WT, Marder SR. The NIMH-MATRICS consensus statement on negative symptoms. Schizophr Bull. (2006) 32:214-9. doi: 10.1093/schbul/sbj053

16. Alpert M, Shaw RJ, Pouget ER, Lim KO. A comparison of clinical ratings with vocal acoustic measures of flat affect and alogia. J Psychiatr Res. (2002) 36:347-53. doi: 10.1016/S0022-3956(02)00016-X

17. Ko YH, Joe SK, Cho W, Park JH, Lee JJ, Jung IK, et al. Estrogen, cognitive function and negative symptoms in female schizophrenia. Neuropsychobiology. (2006) 53:169-75. doi: 10.1159/000093780

18. Moriarty PJ, Lieber D, Bennet A, White L, Parrella M, Harvey PD. Gender differences in poor outcome patients with lifelong schizophrenia. Schizophr Bull. (2001) 27:103-13. doi: 10.1093/oxfordjournals.schbul. a006850
19. Galderisi S, Bucci P, Üçok A, Peuskens J. No gender differences in social outcome in patients suffering from schizophrenia. Eur Psychiatry. (2012) 27:406-8. doi: 10.1016/j.eurpsy.2011.01.011

20. Wójciak P, Domowicz K, Andrzejewska M, Rybakowski JK. Negative symptoms in schizophrenia, assessed by the brief negative symptom scale, selfevaluation of negative symptom scale, and social cognition: a gender effect. Int J Psychiatry Clin Pract. (2021) 25:252-7. doi: 10.1080/13651501.2020.1 810278

21. Mendrek A, Mancini-Marië A. Sex/gender differences in the brain and cognition in schizophrenia. Neurosci Biobehav Rev. (2016) 67:57-78. doi: 10.1016/j.neubiorev.2015.10.013

22. Goldstein JM, Seidman LJ, Goodman JM, Koren D, Lee H, Weintraub S. Are there sex differences in neuropsychological function among patients with schizophrenia? Am J Psychiatry. (1998) 155:1358-64. doi: 10.1176/ajp.155.10.1358

23. Vaskinn A, Sundet K, Simonsen C, Hellvin T, Melle I, Andreassen OA. Sex differences in neuropsychological performance and social functioning in schizophrenia and bipolar disorder. Neuropsychology. (2011) 25:499-510. doi: $10.1037 / \mathrm{a} 0022677$

24. Han M, Huang XF, Chen da C, Xiu MH, Hui L, Liu H, et al. Gender differences in cognitive function of patients with chronic schizophrenia. Neuropsychopharmacol Biol Psychiatry. (2012) 39:358-63. doi: 10.1016/j.pnpbp.2012.07.010

25. Perlick D, Mattis S, Stastny P, Teresi J. Gender differences in cognition in schizophrenia. Schizophr Res. (1992) 8:69-73. doi: 10.1016/0920-9964(92)90062-A

26. Lewine RR, Walker EF, Shurett R, Caudle J, Haden C. Sex differences in neuropsychological functioning among schizophrenic patients. Am J Psychiatry. (1996) 153:1178-80. doi: 10.1176/ajp.153.9.1178

27. Andia AM, Zisook S, Heaton RK, Hesselink J, Jernigan T, Kuck J, et al. Gender differences in schizophrenia. J Nerv Ment Dis. (1995) 183:522-8. doi: 10.1097/00005053-199508000-00005

28. Kao YC, Liu YP, Lien YJ, Lin SJ, Lu CW, Wang TS, et al. The influence of sex cognitive insight and neurocognitive functioning in schizophrenia. Prog Neuropsychopharmacol Biol Psychiatry. (2013) 1:193-200. doi: 10.1016/j.pnpbp.2013.02.006

29. Hoff AL, Kremen WS, Wieneke MH, Lauriello J, Blankfeld HM, Faustman WO. Association of estrogen levels with neuropsychological performance in women with schizophrenia. Am J Psychiatry. (2001) 158:1134-9. doi: 10.1176/appi.ajp.158.7.1134

30. Morgan VA, Castle DJ, Jablensky AV. Do women express and experience psychosis differently from men? Epidemiological evidence from the Australian National Study of Low Prevalence (Psychotic) Disorders. Aust N Z J Psychiatry. (2008) 42:74-82. doi: 10.1080/00048670701732699

31. McEwen BS, Milner TA. Understanding the Broad Influence of Sex Hormones and Sex Differences in the Brain. J Neurosci Res. (2017) 95:24-39. doi: 10.1002/jnr.23809

32. Hajszan T, Milner TA, Leranth C. Sex steroids and the dentate gyrus. Prog Brain Res. (2007) 163:399-415. doi: 10.1016/S0079-6123(07)63023-4

33. Hojo Y. Hattori T-a, Enami T, Furukawa A, Suzuki K, Ishii K, et al. Adult Male rat hippocampus synthesizes estradiol from pregnenolone by cytochromes P45017 $\alpha$ and P450 aromatase localized in neurons. Proc Natl Acad Sci USA. (2004) 101:865-70. doi: 10.1073/pnas.2630225100

34. Li R, Ma X, Wang G, Yang J, Wang Ch. Why sex differences in schizophrenia? J Transl Neurosci. (2016) 1:37-42.

35. Grigoriadis S, Seeman MV. The role of estrogen in schizophrenia: implications for schizophrenia practice guidelines for women. Can J Psychiatry. (2002) 47:437-42. doi: 10.1177/070674370204700504

36. Kaneda Y, Ohmori T. Relation between estradiol and negative symptoms in men with schizophrenia. J Neuropsychiatry Clin Neurosci. (2005) 17:238-42. doi: 10.1176/jnp.17.2.239

37. Fink G, Sumner BE. McQueen JK, Wilson H, Rosie R. Sex steroid control of mood, mental state and memory. Clin Exp Pharmacol Physiol. (1998) 25:764-75. doi: 10.1111/j.1440-1681.1998.tb02151.x

38. Ko YH, Jung SW, Joe SH, Lee HCH, Jung HG, Jung IK. Association between serum testosterone levels and severity of negative symptoms in male patients with chronic schizophrenia. Psychoneuroendocrinology. (2007) 32:385-91. doi: 10.1016/j.psyneuen.2007.02.002 
39. Sisek-Sprem M, Krizaj A, Jukic V, Milosevic M, Petrovic Z, Herceg M. (2015). Testosterone levels and clinical features of schizophrenia with emphasis on negative symptoms and aggression. Nord J Psychiatry. (2015) 69:102-9. doi: $10.3109 / 08039488.2014 .947320$

40. Sasayama D, Hattori K, Teraishi T, Hori H, Ota M, Yoshida S. Negative correlation between cerebrospinal fluid oxytocin levels and negative symptoms of male patients with schizophrenia. Schizophr Res. (2012) 139:2016. doi: 10.1016/j.schres.2012.06.016

41. Frost KH, Keller WR, Buchanan RW, Gold JM, Koenig JI, Ossenfort KL. Plasma Oxytocin Levels are Associated with Impaired Social Cognition and Neurocognition in Schizophrenia. Archives of Clinical Neuropsycholog. (2014) 29:577-8. doi: 10.1093/arclin/acu038.195

42. Feitel D. Is oxytocin a promising treatment for schizophrenia? Expert Rev Neurother. (2011) 11:157-9. doi: 10.1586/ern.10.199

43. Kirkpatrick B, Strauss GP, Nguyen L, Fischer BA, Daniel DG, Cienfuegos A. The brief negative symptom scale: psychometric properties. Schizophr Bull. (2011) 37:300-5. doi: 10.1093/schbul/sbq059

44. Dollfus S, Mach C, Morello R. Self-evaluation of negative symptoms: a novel tool to assess negative symptoms. Schizophr Bull. (2016) 42:571-8. doi: $10.1093 / \mathrm{schbul} / \mathrm{sbv} 161$

45. World Medical Association General Assembly. Declaration of Helsinki, Ethical Principles for Medical Research Involving Human Subjects. Fortaleza: World medical Association (2013)

46. Kay SR, Fiszbein A, Opler LA. The positive and negative syndrome scale (PANSS) for schizophrenia. Schizophr Bull. (1987) 13:261-76. doi: 10.1093/schbul/13.2.261

47. Wójciak P, Górna K, Domowicz K, Jaracz K, Gołebiewska K, Michalak M. Polish version of the Brief Negative Symptom Scale (BNSS). Psychiatr Pol. (2019) 53:541-9. doi: 10.12740/PP/OnlineFirst/91490

48. Wójciak P, Górna K, Domowicz K, Jaracz K, Szpalik R, Michalak M. Polish version of the Self-evaluation of Negative Symptoms (SNS). Psychiatr Pol. (2019) 53:551-9. doi: 10.12740/PP/OnlineFirst/97352

49. Morosini PL, Magliano L, Brambilla L, Ugolini S, Pioli R. Development, reliability and acceptability of a new version of the DSM-IV Social and Occupational Functioning Assessment Scale (SOFAS) to assess routine social functioning. Acta Psychiatr Scand. (2000) 101:323-9. doi: 10.1111/j.1600-0447.2000.tb10933.x

50. Reitan RM. Validity of the Trail Making Test as an indicator of organic brain damage. Percept Mot Skills. (1958) 8:271-6. doi: 10.2466/pms.1958.8.3.271

51. Periáñez JA, Ríos-Lago M, Rodríguez-Sánchez JM, Adrover-Roig D, SánchezCubillo I, Crespo-Facorro B. Trail Making Test in traumatic brain injury, schizophrenia, and normal ageing: Sample comparisons and normative data. Arch Clin Neuropsychol. (2007) 22:433-47. doi: 10.1016/j.acn.2007. 01.022

52. Stroop JR. Studies of interference in serial verbal reaction. J Exp Psychology. (1935) 18:643-62. doi: 10.1037/h0054651

53. Tyburski E, Karabanowicz E, Mak M, Lebiecka Z, Samochowiec A, PełkaWysiecka J. Color trails test: a new set of data on cognitive flexibility and processing speed in schizophrenia. Front Psychiatry. (2020) 11:521. doi: 10.3389/fpsyt.2020.00521

54. Thurstone LL. Primary Mental Abilities. Chicago: University of Chicago Press (1938).
55. Piskunowicz M, Bieliński M, Zgliński A, Borkowska A. Verbal fluency tests-application in neuropsychological assessment. Psychiatria Polska. (2013) 47: 475-85.

56. Wechsler D. Manual for the Wechsler Adult Intelligence Scale. New York: The Psychological Corporation (1955).

57. Brzeziński J, Gaul M, Hornowska E, Machowski A, Zakrzewska MS. Wechslera dla dorostych Wersja zrewidowana WAIS-R (PL). Warszawa: Pracownia Testów Psychologicznych PTP (1996).

58. Wójciak P, Domowicz K, Rybakowski JK. Metabolic indices in schizophrenia: association of negative symptoms with higher HDL cholesterol in female patients. World J Biol Psychiatry. (2021) 22:552-6. doi: 10.1080/15622975.2020.1849796

59. García-Portilla MP, García-Álvarez L, González-Blanco L, Dal Santo F, BobesBascarán T, Martínez-Cao C, et al. Real-world functioning in patients with schizophrenia: beyond negative and cognitive symptoms. Front Psychiatry. (2021) 12:700747. doi: 10.3389/fpsyt.2021.700747

60. Priyamvada R, Ranjan R, Jha GK, Chaudhury S. Correlation of neurocognitive deficits with positive and negative symptoms in schizophrenia. Ind Psychiatry J. (2021) 30:249-54. doi: 10.4103/ipj.ipj_44_20

61. Jeakal E, Park K, Lee E, Strauss GP, Choi KH. Validation of the Brief Negative Symptom Scale in Korean patients with schizophrenia. Asia Pac Psychiatry. (2020) 12:e12382. doi: 10.1111/appy.12382

62. Eack SM, Keshavan MS. Cognition, negative symptoms, and functional outcome in psychosis. Schizophr Res. (2020) 224:22-3. doi: 10.1016/j.schres.2020.06.029

63. Luther L, Suor JH, Rosen C, Jobe TH, Faull RN, Harrow M. Clarifying the direction of impact of negative symptoms and neurocognition on prospective work functioning in psychosis: a 20-year longitudinal study. Schizophr Res. (2020) 220:232-9. doi: 10.1016/j.schres.2020.03.012

64. Bartholomeusz CF, Allott K. Neurocognitive and social cognitive approaches for improving functional outcome in early psychosis: theoretical consideration and current state of evidence. Schizophrenia Res Treatment. (2012) 2012:815315. doi: 10.1155/2012/815315

Conflict of Interest: The authors declare that the research was conducted in the absence of any commercial or financial relationships that could be construed as a potential conflict of interest.

Publisher's Note: All claims expressed in this article are solely those of the authors and do not necessarily represent those of their affiliated organizations, or those of the publisher, the editors and the reviewers. Any product that may be evaluated in this article, or claim that may be made by its manufacturer, is not guaranteed or endorsed by the publisher.

Copyright (c) 2021 Wójciak, Domowicz, Zabłocka, Michalak and Rybakowski. This is an open-access article distributed under the terms of the Creative Commons Attribution License (CC BY). The use, distribution or reproduction in other forums is permitted, provided the original author(s) and the copyright owner(s) are credited and that the original publication in this journal is cited, in accordance with accepted academic practice. No use, distribution or reproduction is permitted which does not comply with these terms. 